\title{
7. ACTIONS WITH BOREL ORBIT EQUIVALENCE RELATIONS
}

If $G$ is a Polish group and $(X, a)$ a Borel $G$-space, then the associated equivalence relation $E_{a}$ is analytic but in general not Borel, even when $X$ is a Polish $G$-space (see 3.2). We study in this section various questions concerning the Borelness of such $E_{a}$.

\subsection{Characterizations}

We first provide a number of equivalent conditions characterizing when $E_{a}$ is Borel. The next result is well-known (see Sami $[94,3.6]$ ).

7.1.1 Theorem. (Sami [94]) Let $G$ be a Polish group and $(X, a)$ a Polish $G$-space. Then $E_{a}$ is Borel iff there is $\alpha<\omega_{1}$ so that all the orbits $G \cdot x$ are $\Pi_{\alpha}^{0}$.

Proof. For the nontrivial direction, fix such an $\alpha$. Then

$$
x E_{a} y \Leftrightarrow x E_{a}^{\alpha} y,
$$

where

$$
x E_{a}^{\alpha} y \Leftrightarrow \text { for all } \Pi_{\alpha}^{0} \text { invariant } A \subseteq X, x \in A \Leftrightarrow y \in A .
$$

As shown in Sami [94, 3.7], $E_{a}^{\alpha}$ is $\boldsymbol{\Pi}_{1}^{1}$, so $E_{a}$ is both analytic and co-analytic, thus Borel.

The second equivalent formulation relates the complexity of $E_{a}$ to that of the function $x \mapsto G_{x}$, assigning to each point of $X$ its stabilizer. Let also, for $x, y \in X, G_{x, y}$ be the set $\{g \in G: g \cdot x=y\}=h G_{x}$ for any $h \in G$, with $h \cdot x=y$. We have:

7.1.2 Theorem. Let $G$ be a Polish group and $(X, a)$ a Borel $G$-space. Then the following are equivalent:

(i) $E_{a}$ is Borel.

(ii) The map $x \mapsto G_{x}$ from $X$ into $\mathcal{F}(G)$ is Borel.

(iii) The map $(x, y) \mapsto G_{x, y}$ from $X^{2}$ into $\mathcal{F}(G)$ is Borel. 
Proof. (ii) $\Rightarrow$ (i) is well-known and easily follows from 1.2.4. Indeed, let $T$ be as in the statement of 1.2.4. Then

$$
\begin{aligned}
x E_{a} y & \Leftrightarrow \exists g \in G(g \cdot x=y) \\
& \Leftrightarrow \exists ! g\left(g \in T_{G_{x}} \& g \cdot x=y\right) \\
& \Leftrightarrow \exists ! g\left[\left(g, G_{x}\right) \in T \& g \cdot x=y\right],
\end{aligned}
$$

so $E_{a}$ is analytic and co-analytic, thus Borel.

(i) $\Rightarrow$ (ii). Assume now that $E_{a}$ is Borel in order to show that $x \mapsto G_{x}$ is Borel. Fix a basis $\left\{U_{n}\right\}$ of nonempty open sets in $G$, with $G \in\left\{U_{n}\right\}$.

The following lemma can be proved by standard methods.

7.1.3 Lemma. There is a Borel set $\mathcal{B} \subseteq X \times \mathbb{N} \times X$ such that, letting $\mathcal{B}_{x, n}=\{y:(x, n, y) \in \mathcal{B}\}$ and

$$
\mathcal{B}(x)=\left\{\mathcal{B}_{x, n}: n \in \mathbb{N}\right\}
$$

we have:

(a) $G \cdot x \in \mathcal{B}(x)$;

(b) $B \in \mathcal{B}(x) \Rightarrow B^{\Delta U_{n}} \in \mathcal{B}(x)$;

(c) $\mathcal{B}(x)$ is a Boolean algebra;

(d) the topology $t^{\prime}(x)$ generated by $\mathcal{B}(x)$ is Polish.

Proof. For every fixed $x$ we can find a countable sequence $\mathcal{B}_{x, n}$ of Borel sets so that if $\mathcal{B}(x)=\left\{\mathcal{B}_{x, n}: n \in \mathbb{N}\right\}$, then (a) - (d) are satisfied. This easily follows from 5.1.1, 5.1.3 (b) and 5.1.7 (h). The uniformity, i.e., the fact that

$$
\mathcal{B}(x, n, y) \Leftrightarrow y \in \mathcal{B}(x, n)
$$

is Borel, is implicit in the proof of these results, using the fact that not only each orbit $G \cdot x$ is Borel (which is always the case) but according to our basic hypothesis, this holds uniformly, i.e., $\{(x, y): y \in G \cdot x\}=\left\{(x, y): x E_{a} y\right\}$ is Borel.

It now follows from the proof of 5.2.1 that if $t(x) \subseteq t^{\prime}(x)$ is the topology generated by $\left\{B^{\Delta U_{n}}: B \in \mathcal{B}(x), n \in \mathbb{N}\right\}$, then $(X, t(x))$ is Polish and the action is continuous for $(X, t(x))$. Moreover, since $G \cdot x=(G \cdot x)^{\Delta G}$ and $X \backslash G \cdot x=(X \backslash G \cdot x)^{\Delta G}$, clearly $G \cdot x$ is clopen in $(X, t(x))$. It then follows from Effros' Theorem 2.2.2 that the canonical map $g G_{x} \mapsto g \cdot x$ is a homeomorphism of $G / G_{x}$ onto $G \cdot x$ (with the relative $t(x)$-topology). Thus, if $U \subseteq G$ is open, then $U \cdot x$ is open in $t(x)$. 
To show that $x \mapsto G_{x}$ is Borel it is necessary and sufficient to show that for each open $U \subseteq G$ the set

$$
\left\{x: G_{x} \cap U \neq \emptyset\right\}
$$

is Borel. This is easily equivalent to showing that for each open nonempty $U, V \subseteq G$ the set

$$
\left\{x: U G_{x} \cap V \neq \emptyset\right\}
$$

is Borel. This in turn immediately follows from the following claim:

Claim.

$$
\begin{aligned}
& U G_{x} \cap V \neq \emptyset \Leftrightarrow \exists U_{m} \subseteq U \forall B \in \mathcal{B}(x)\left(x \in B^{\Delta U_{m}} \Rightarrow x \in B^{\Delta V}\right) \\
&\left(\Leftrightarrow \exists m \forall n\left(U_{m} \subseteq U \&\left(x \in B_{x, n}^{\Delta U_{m}} \Rightarrow x \in B_{x, n}^{\Delta V}\right)\right)\right) .
\end{aligned}
$$

Proof of claim. $\Rightarrow$ : If $U G_{x} \cap V \neq \emptyset$, there is $g \in G_{x}$ and $U_{m} \subseteq U$ with $U_{m} g \subseteq V$. Fix $B \in \mathcal{B}(x)$ such that $x \in B^{\Delta U_{m}}=B^{\Delta\left(\left(U_{m} g\right) g^{-1}\right)}$. Then $g^{-1} \cdot x \in B^{\Delta\left(U_{m} g\right)}$. But $g^{-1} \cdot x=x$ and $U_{m} g \subseteq V$, so $x \in B^{\Delta V}$.

$\Leftarrow$ : Assume that $U G_{x} \cap V=\emptyset$ and fix $U_{m} \subseteq U$. Then $U \cdot x \cap V \cdot x=\emptyset$, so $U_{m} \cdot x \cap V \cdot x=\emptyset$. Since $U_{m} \cdot x$ is open in $t(x)$, there is a nonempty $B \in \mathcal{B}(x)$ such that $B$ is open in $t(x)$ and $B \subseteq U_{m} \cdot x$. Then $\{g: g \cdot x \in B\}$ is open in $G$ and it intersects $U_{m}$. So let $U_{k} \subseteq\{g: g \cdot x \in B\} \cap U_{m}$. Then if $g \in U_{k}$ we have that $g \cdot x \in B$, so $x \in B^{\Delta U_{m}}$. On the other hand, $x \notin B^{\Delta V}$ (since otherwise there is $g \in V$ with $g \cdot x \in B$, so $B \cap V \cdot x \neq \emptyset$, a contradiction).

(iii) $\Rightarrow$ (ii) is obvious since $G_{x}=G_{x, x}$.

(ii) $\Rightarrow$ (iii): Let $T$ be as in the statement of 1.2.4. Then for $U \neq \emptyset$ open in $G$ and $\left\{U_{n}\right\}$ as before we have:

$$
\begin{aligned}
G_{x, y} \cap U \neq \emptyset & \Leftrightarrow \exists g \in U(g \cdot x=y) \\
& \Leftrightarrow \exists ! h \in T_{G_{x}}\left[h \cdot x=y \& \exists g^{\prime} \in G_{x}\left(h g^{\prime} \in U\right)\right] \\
& \Leftrightarrow \exists ! h\left[\left(h, G_{x}\right) \in T \& h \cdot x=y \& G_{x} \cap h^{-1} U \neq \emptyset\right] .
\end{aligned}
$$

Since

$$
\begin{aligned}
R(x, h) & \Leftrightarrow G_{x} \cap h^{-1} U \neq \emptyset \\
& \Leftrightarrow \exists n\left[U_{n} \cap G_{x} \neq \emptyset \& h U_{n} \subseteq U\right]
\end{aligned}
$$

is both analytic and co-analytic, thus Borel, it follows that $\left\{(x, y): G_{x, y} \cap\right.$ $U \neq \emptyset\}$ is both analytic and co-analytic, thus Borel.

We now apply 7.1 .2 to the special case of $S_{\infty}$ and the logic action $J_{L}$. The stabilizer $G_{x}$ of any $x \in X_{L}$ is just the automorphism group $\operatorname{Aut}\left(\mathcal{A}_{x}\right)$ of the structure coded by $x$. 
Definition. For any countable structure $\mathcal{A}=(A,-)$ of $L$ and tuples $\bar{a}, \bar{b}$ of the same length from $A$ define inductively

$$
(\mathcal{A}, \bar{a}) \equiv^{\alpha}(\mathcal{A}, \bar{b})
$$

as follows:

$$
(\mathcal{A}, \bar{a}) \equiv^{0}(\mathcal{A}, \bar{b})
$$

iff for any atomic formula $\varphi$,

$$
\begin{gathered}
\mathcal{A} \models \varphi[\bar{a}] \Leftrightarrow \mathcal{A} \models \varphi[\bar{b}] ; \\
(\mathcal{A}, \bar{a}) \equiv^{\alpha+1}(\mathcal{A}, \bar{a})
\end{gathered}
$$

iff

$$
\begin{aligned}
& \forall c \in A \exists d \in A\left[(\mathcal{A}, \bar{a}, c) \equiv^{\alpha}(\mathcal{A}, \bar{b}, d)\right] \& \\
& \forall d \in A \exists c \in A\left[(\mathcal{A}, \bar{a}, c) \equiv^{\alpha}(\mathcal{A}, \bar{b}, d)\right] \\
& (\mathcal{A}, \bar{a}) \equiv^{\lambda}(\mathcal{A}, \bar{b}) \Leftrightarrow \forall \alpha<\lambda(\mathcal{A}, \bar{a}) \equiv^{\alpha}(\mathcal{A}, \bar{b}),
\end{aligned}
$$

when $\lambda$ is limit. There is a least (countable) ordinal $\alpha$ such that for all $\beta \geq \alpha$, all $n$ and all $\bar{a}, \bar{b} \in A^{n}$, we have

$$
(\mathcal{A}, \bar{a}) \equiv^{\beta}(\mathcal{A}, \bar{b}) \Leftrightarrow(\mathcal{A}, \bar{a}) \equiv^{\alpha}(\mathcal{A}, \bar{b}) .
$$

It is called the $S$ cott rank of $\mathcal{A}$, in symbols $\operatorname{sr}(\mathcal{A})$. Moreover

$$
\begin{aligned}
(\mathcal{A}, \bar{a}) \equiv^{s r(\mathcal{A})}(\mathcal{A}, \bar{b}) & \Leftrightarrow \exists \pi \in \operatorname{Aut}(\mathcal{A})(\pi(\bar{a})=\bar{b}) \\
& \Leftrightarrow(\mathcal{A}, \bar{a}) \cong(\mathcal{A}, \bar{b}) .
\end{aligned}
$$

The quantifier-rank of an $L_{\omega_{1} \omega}$ formula $\varphi$, denoted $q r(\varphi)$, is a countable ordinal, defined by induction on formulas as follows:

(a) $\varphi$ atomic $\Rightarrow q r(\varphi)=0$.

(b) $\operatorname{qr}(\neg \varphi)=\operatorname{qr}(\varphi)$.

(c) $\operatorname{qr}(\forall v \varphi)=q r(\exists v \varphi)=q r(\varphi)+1$.

(d) $q r\left(\bigwedge_{n \in \mathbb{N}} \varphi_{n}\right)=q r\left(\bigvee_{n \in \mathbb{N}} \varphi_{n}\right)=\sup _{n}\left(q r\left(\varphi_{n}\right)\right)$.

It is easy to see that $\mathcal{A} \equiv^{\alpha} \mathcal{B}$ iff for any $L_{\omega_{1} \omega}$ sentence $\varphi$ such that $\operatorname{qr}(\varphi) \leq \alpha, \mathcal{A} \models \varphi \Leftrightarrow \mathcal{B} \models \varphi$, i.e., $\mathcal{A}$ and $\mathcal{B}$ agree about the truth of all sentences of quantifier rank $\leq \alpha$. 
Recall that the invariant Borel sets of $X_{L}$ are exactly the sets of the form

$$
\operatorname{Mod}(\sigma)=\left\{x \in X_{L}: \mathcal{A}_{x} \vDash \sigma\right\},
$$

for some $L_{\omega_{1} \omega}$ sentence $\sigma$. In particular, since by 2.3 .3 every orbit $\{y \in$ $\left.X_{L}: \mathcal{A}_{x} \cong \mathcal{A}_{y}\right\}$ is Borel, we obtain Scott's theorem that for any countable $L$-structure $\mathcal{A}$ there is an $L_{\omega_{1} \omega}$ sentence $\sigma$ such that for countable $\mathcal{B}, \mathcal{B} \cong$ $\mathcal{A} \Leftrightarrow \mathcal{B} \models \sigma$. Such a sentence is called a Scott sentence for $\mathcal{A}$.

There is actually a canonical way to construct such a sentence for $\mathcal{A}$, called the canonical Scott sentence $\sigma_{\mathcal{A}}$. The transfinite Scott analysis of countable structures leading to the construction of the canonical Scott sentence can be found, e.g., in Barwise [75]. This is similar to the analysis used to define Scott rank, above. And there is a relationship:

$$
q r\left(\sigma_{\mathcal{A}}\right)=\operatorname{sr}(\mathcal{A})+\omega
$$

7.1.4 Corollary. Let $L$ be a (countable relational) language and $\sigma$ an $L_{\omega_{1} \omega}$ sentence. The following are equivalent:

(i) The set

$$
\left\{(x, y) \in \operatorname{Mod}(\sigma)^{2}: \mathcal{A}_{x} \cong \mathcal{A}_{y}\right\}
$$

is Borel.

(ii) The set

$$
\left\{(x, y, \bar{a}, \bar{b}) \in \operatorname{Mod}(\sigma)^{2} \times \bigcup_{n}\left(\mathbb{N}^{n}\right)^{2}:\left(\mathcal{A}_{x}, \bar{a}\right) \cong\left(\mathcal{A}_{y}, \bar{b}\right)\right\}
$$

is Borel. (Here $\bigcup_{n}\left(\mathbb{N}^{n}\right)^{2}$ is given the discrete topology.)

(iii) The set

$$
\left\{(x, \bar{a}, \bar{b}) \in \operatorname{Mod}(\sigma) \times \bigcup_{n}\left(\mathbb{N}^{n}\right)^{2}:\left(\mathcal{A}_{x}, \bar{a}\right) \cong\left(\mathcal{A}_{x}, \bar{b}\right)\right\}
$$

is Borel.

(iv) There is $\alpha<\omega_{1}$ such that

$$
\operatorname{sr}(\mathcal{A}) \leq \alpha
$$

for any countable model $\mathcal{A}$ of $\sigma$.

Proof. The equivalence of (i), (ii) and (iii) is a straightforward consequence of 7.1.2 for the logic action of $S_{\infty}$ on $\operatorname{Mod}(\sigma)$, using the standard basis $\left\{N_{b}\right\}$ 
of $S_{\infty}$ as in 1.5. The implication (iv) $\Rightarrow$ iii) is obvious, while the implication (iii) $\Rightarrow$ (iv) follows by a standard boundedness argument. For example, from a result of Nadel [74] (see also Barwise [75, 7.4]) and assuming that $L$ is recursive (otherwise we relativize), it follows that $\operatorname{sr}\left(\mathcal{A}_{x}\right) \leq \omega_{1}^{x}=$ the first ordinal not recursive in $x$. Thus if $\left(\mathcal{A}_{x}, \bar{a}\right) \not \nexists\left(\mathcal{A}_{x}, \bar{b}\right)$, where $x \in \operatorname{Mod}(\sigma)$, it follows that there is $\alpha<\omega_{1}^{x}$ with $\left(\mathcal{A}_{x}, \bar{a}\right) \not^{\alpha}\left(\mathcal{A}_{x}, \bar{b}\right)$. Since the set in (iii) is Borel, it follows, by boundedness, that there is a fixed ordinal $\alpha<\omega_{1}$ such that for any $\left(\mathcal{A}_{x}, \bar{a}\right) \not\left(\mathcal{A}_{x}, \bar{b}\right)$, with $x \in \operatorname{Mod}(\sigma)$, we have that $\left(\mathcal{A}_{x}, \bar{a}\right) \not^{\alpha}\left(\mathcal{A}_{x}, \bar{b}\right)$, so for any $x \in \operatorname{Mod}(\sigma), \operatorname{sr}\left(\mathcal{A}_{x}\right) \leq \alpha$ and (iv) holds. $\dashv$

Remark. We have originally proved 7.1.4 using (among other things) a result of Nadel [74, 5.1], which implies that if some Scott sentence of a countable structure belongs to a countable admissible set, then the Scott rank of the structure is smaller than the ordinal of the admissible set. Nadel's proof used the omitting types theorem for fragments of $L_{\omega_{1} \omega}$. Our proof of Theorem 7.1.2 has been motivated, in a sense that is hard to explain precisely, by Nadel's proof. (The Effros Theorem, for example, is used as a replacement of the omitting types argument.)

\subsection{Some effective considerations}

One can also obtain an effective version of 7.1 .2 with the same proof.

Let $G$ be a recursively presented Polish group with multiplication and inverse recursive, and $Y$ a recursively presented Polish space, which for convenience we assume is perfect, so that it fits in the framework of Moschovakis [80]. Let $X \subseteq Y$ be a $\Delta_{1}^{1}$ subset of $Y$ and let $a$ be an action of $G$ on $X$ which is also $\Delta_{1}^{1}$. We will refer to this as an effective Borel $G$-space. We will fix a canonical basis $\left\{U_{n}\right\}$ for $G$ as in Moschovakis [80, 3.B].

7.2.1 Corollary. If $X$ is an effective Borel $G$-space, then for any $x \in X$ the following are equivalent:

(i) $G \cdot x \in \Delta_{1}^{1}(x)$.

(ii) $\left\{n: G_{x} \cap U_{n} \neq \emptyset\right\} \in \Delta_{1}^{1}(x)$.

From this it follows that for any closed $\Delta_{1}^{1}$ subgroup $F \subseteq G$, the following are equivalent:

(i) $\left\{n: F \cap U_{n} \neq \emptyset\right\}$ is $\Delta_{1}^{1}$;

(ii) There is a $\Delta_{1}^{1}$ transversal for the left-cosets of $F$.

The fact that (i) $\Rightarrow$ (ii) is just the effective version of 1.2.4, and (ii) $\Rightarrow$ (i) follows by applying 7.2.1 to the obvious action of $G$ on a $\Delta_{1}^{1}$ transversal (which we can essentially identify with $G / F$ ). 
Finally, as in Sami $[94, \S 3]$ let

$$
\omega_{1}^{G \cdot x}=\inf \left\{\omega_{1}^{y}: y \in G \cdot x\right\}
$$

where $\omega_{1}^{y}=$ the least ordinal not recursive in $y$. By Sami $[94,3.5], G \cdot x \in$ $\Pi_{\omega_{1}^{G \cdot x}+2}^{0}$. We then have the following fact (also using 7.1.1):

7.2.2 Proposition. If $(X, a)$ is an effective Borel $G$-space, and for some $\alpha<\omega_{1}$ and all $x \in X, \omega_{1}^{G \cdot x} \leq \alpha$, then $E_{a}$ is Borel.

Remark. The converse of 7.2 .2 clearly fails, as we can see by considering the trivial action $g \cdot x=x$.

Let us next make a couple of comments concerning the Topological Vaught Conjecture. By using Silver's Theorem 3.3.1 and the proof of 7.1.1 it is clear that TVC2 $(G)$ (see 6.2) has an equivalent formulation where the conclusion from "countably many or perfectly many orbits in $B$ " is changed to "all the orbits contained in $B$ are $\Pi_{\alpha}^{0}$ for some fixed $\alpha<\omega_{1}$ or else there are perfectly many orbits in $B$ ".

Also in the case of an effective Borel $G$-space $X$, TVC3 (see again 6.2) has the following equivalent formulation: Either $\omega_{1}^{G \cdot x} \leq \alpha$ for all $x \in X$ and some fixed $\alpha<\omega_{1}$ or else there are perfectly many orbits. It follows that for such an action, TVC3 is a $\Sigma_{2}^{1}$ statement. This is because the alternative "there are perfectly many orbits" is clearly $\Sigma_{2}^{1}$, while the statement that " $\exists \alpha<\omega_{1} \forall x\left(\omega_{1}^{G \cdot x} \leq \alpha\right)$ " can be rewritten (using Sami $[94,3.2]$ ) as $\exists \alpha<$ $\omega_{1} \forall x\left(x \in B_{\alpha}^{* G}\right)$, where $B_{\alpha}=\left\{x \in X: \omega_{1}^{x} \leq \alpha\right\}$, and this is clearly $\Sigma_{2}^{1}$ too.

By relativization, TVC2 for any fixed (perfect) Polish $G$-space $X$ and any invariant Borel set $B \subseteq X$ will be $\Sigma_{2}^{1}(p)$, where $p$ is any parameter that encodes $G, X, B$ and the action, so that this becomes an effective Borel $G$-space relative to $p$. In particular, it follows that

TVC is a $\Pi_{3}^{1}$ statement.

As a special case, the Vaught Conjecture VC2 for a given first-order theory $T$ is $\Sigma_{2}^{1}$ in the parameter $T$ and VC2 (for all $T$ ) is a $\Pi_{3}^{1}$ statement. Thus VC2 for a given $T$ is absolute, while it is open whether it is possible that VC2 holds in the constructible universe but fails in a generic extension of it.

\subsection{Decompositions}

We will next prove that one can decompose any Borel $G$-space into $\aleph_{1}$ Borel invariant sets on which the orbit equivalence relation is Borel. 
7.3.1 Theorem. Let $G$ be a Polish group and $(X, a)$ a Borel $G$-space. There is a sequence $\left\{A_{\xi}\right\}_{\xi<\omega_{1}}$ of pairwise disjoint Borel subsets of $X$ such that:

(i) $A_{\xi}$ is invariant and $\bigcup_{\xi} A_{\xi}=X$;

(ii) $E_{a} \mid A_{\xi}$ is Borel;

(iii) there is a $C$-measurable function $f: X \rightarrow 2^{\mathbb{N} \times \mathbb{N}}$ such that $f(x) \in$ WO and $x \in A_{|f(x)|}$, where WO is the set of codes of countable ordinals, and for $w \in \mathrm{WO},|w|=$ the ordinal coded by $w$;

(iv) (boundedness) if $A \subseteq X$ is invariant Borel and $E_{a} \mid A$ is Borel, then $A \subseteq \bigcup_{\xi<\alpha} A_{\xi}$ for some $\alpha<\omega_{1}$.

Remark. Theorem 3.4.4 can be proved quite easily from Theorems 3.4.3 and 7.3.1, by using generic codes for countable ordinals.

Proof (of 7.3.1.) Let $\mathcal{F}(G)$ be the standard Borel space of closed subsets of $G$ with the Effros Borel structure and $\mathcal{S}(G)$ the set of closed subgroups of $G$, which is a Borel set in $\mathcal{F}(G)$. Consider the conjugation action of $G$ on $\mathcal{S}(G)$ :

$$
g \cdot F=g F g^{-1} .
$$

It is Borel (see 2.4, Ex. (ii)). Let now

$$
P=\left\{\left(x, G_{x}\right): x \in X\right\} \subseteq X \times \mathcal{S}(G) .
$$

Then $P$ is $\Pi_{1}^{1}$, since

$$
\begin{aligned}
(x, F) \in P & \Leftrightarrow F=G_{x} \\
& \Leftrightarrow \forall g(g \in F \Leftrightarrow g \cdot x=x) .
\end{aligned}
$$

Next consider the product action of $G$ on $X \times \mathcal{S}(G)$ :

$$
g \cdot(x, F)=\left(g \cdot x, g F g^{-1}\right) .
$$

Since $g \cdot\left(x, G_{x}\right)=\left(g \cdot x, g G_{x} g^{-1}\right)=\left(g \cdot x, G_{g \cdot x}\right), P$ is invariant under this action. By a result of Solovay (see, e.g., Kechris $[95,34.6]$ ) there is a $\boldsymbol{\Pi}_{1}^{1}$ rank $\varphi: P \rightarrow \omega_{1}$ which is also invariant under this action. Moreover, there is a $C$-measurable function $p: X \times \mathcal{S}(G) \rightarrow$ WO with $\varphi\left(x, G_{x}\right)=\left|p\left(x, G_{x}\right)\right|$. Put

$$
P_{\xi}=\left\{\left(x, G_{x}\right) \in P: \varphi\left(x, G_{x}\right)=\xi\right\},
$$

and

$$
A_{\xi}=\operatorname{proj}_{X}\left(P_{\xi}\right)=\left\{x:\left(x, G_{x}\right) \in P_{\xi}\right\}
$$


Since $P_{\xi}$ is Borel and the graph of a function, $A_{\xi}$ is clearly Borel. It is also invariant. Since also $x \mapsto G_{x}$ is Borel on $A_{\xi}$ (having graph $P_{\xi}$ ), $E_{a} \mid P_{\xi}$ is Borel by 7.1.2. So (i), (ii) hold. Next, the function $x \mapsto G_{x}$ from $X$ into $\mathcal{S}(G)$ is $C$-measurable, since for open $U \subseteq G$ the set

$$
\mathcal{U}(x) \Leftrightarrow G_{x} \cap U \neq \emptyset \Leftrightarrow \exists g(g \in U \& g \cdot x=x)
$$

is analytic. Letting $f(x)=p\left(x, G_{x}\right)$, we have that (iii) holds.

Finally, if $A \subseteq X$ is invariant Borel and $E_{a} \mid A$ is Borel, then, by 7.1.2 again, the map $x \mapsto G_{x}$ is Borel on $A$, thus $\left\{\left(x, G_{x}\right): x \in A\right\}$ is a $\Sigma_{1}^{1}$ subset of $P$, so by the boundedness theorem for $\Pi_{1}^{1}$-ranks (see, e.g., Kechris [95, 35.23]), there is $\alpha<\omega_{1}$ with $\varphi\left(x, G_{x}\right)<\alpha$ for all $x \in A$, i.e., $A \subseteq \bigcup_{\xi<\alpha} A_{\xi}$.

Remarks. (a) Assuming we have an effective Borel $G$-action (otherwise we relativize), we can use the ordinals $\omega_{1}^{G \cdot x}$ as in 7.2 to define $A_{\xi}=\{x \in$ $\left.X: \omega_{1}^{G \cdot x}=\xi\right\}$. This gives a different decomposition satisfying (i), (ii), (iii) but not necessarily (iv), as we can see by again considering the trivial action $g \cdot x=x$. (b) In the case of logic actions, Theorem 7.3.1 (i) - (iii) is well-known. One can take $A_{\xi}=\left\{x \in X_{L}: \operatorname{sr}\left(\mathcal{A}_{x}\right)+\omega=\xi\right\}$, and then isomorphism restricted to $A_{\xi}$ is $\equiv \xi$. Part (iv) of 7.3 .1 then follows from 7.1.4.

The following is immediate from 7.3.1, (iv).

7.3.2 Corollary. Let $G$ be a Polish group and $\left(\mathcal{U}_{G}, u_{G}\right)$ a universal Borel $G$-space. Let $\left\{A_{\xi}\right\}$ be a decomposition of $\mathcal{U}_{G}$ as in 7.3.1. Put

$$
F_{\alpha}^{G}=E_{u_{G}} \mid \bigcup_{\xi<\alpha} A_{\xi}
$$

Then $\left\{F_{\alpha}^{G}\right\}$ is a universal family for the class

$$
\mathcal{E}_{G}^{B}=\left\{E_{a}:(X, a) \text { is a Borel } G \text {-space with } E_{a} \text { Borel }\right\},
$$

i.e., for every $E \in \mathcal{E}_{G}^{B}$, there is $\alpha$ with $E$ e.r.-embeddable into $F_{\alpha}^{G}$.

We can also obtain a global version of 7.3.2. We first need the following lemma.

7.3.3 Lemma. In the notation of 2.3.5, if $E_{a}$ is Borel, so is $E_{b}$.

Proof. Assume $E_{a}$ is Borel, so $x \mapsto G_{x}$ is Borel, by 7.1.2. It is enough to show (by 7.1.2 again) that the map $y \in Y \mapsto H_{y}$ is Borel. But if $y=[x, h]$, 
then $H_{[x, h]}=H_{b\left(h^{-1},[x, 1]\right)}=h^{-1} H_{[x, 1]} h$. Now

$$
\begin{aligned}
h \in H_{[x, 1]} & \Leftrightarrow b(h,[x, 1])=[x, 1] \\
& \Leftrightarrow\left[x, h^{-1}\right]=[x, 1] \\
& \Leftrightarrow \exists g \in G\left(g \cdot x=x \& g h^{-1}=1\right) \\
& \Leftrightarrow h \in G \& h \cdot x=x \\
& \Leftrightarrow h \in G_{x} .
\end{aligned}
$$

So $H_{[x, h]}=h^{-1} G_{x} h$. Thus for $F \in \mathcal{F}(H), y \in Y$,

$$
F=H_{y} \Leftrightarrow \exists x \in X \exists h \in H\left([x, h]=y \& F=h^{-1} G_{x} h\right) .
$$

Since the map $(x, h) \in X \times H \mapsto[x, h] \in Y$ is Borel, this shows that the graph of the map $y \mapsto H_{y}$ is analytic, thus this map is Borel, and the proof is complete.

7.3.4 Corollary. Let $(\mathcal{U}, u)$ be a universal Borel $H\left(I^{\mathbb{N}}\right)$-space and let $\left\{A_{\xi}\right\}_{\xi<\omega_{1}}$ be a decomposition as in 7.3.1. Put

$$
F_{\alpha}=E_{u} \mid \bigcup_{\xi<\alpha} A_{\xi}
$$

Then $\left\{F_{\alpha}\right\}$ is a universal family for the class

$$
\begin{gathered}
\mathcal{E}^{B}=\left\{E_{a}:(X, a) \text { is a Borel } G \text {-space for some Polish group } G\right. \\
\text { and } \left.E_{a} \text { is Borel }\right\}
\end{gathered}
$$

i.e., for every $E \in \mathcal{E}^{B}$, there is an $\alpha$ with $E$ e.r.-embeddable into $F_{\alpha}$.

Proof. By 1.4.1, 7.3.3 and 7.3.2.

In the case $G=S_{\infty}$ we can describe explicitly $\left\{F_{\alpha}^{S_{\infty}}\right\}$. Consider the language $L_{0}=\{\in\}$ of set theory and let

$$
\begin{aligned}
A_{\xi}= & \left\{x \in X_{L_{0}}:\left(\mathcal{A}_{x}^{L_{0}}=\left(\mathbb{N}, \in_{x}\right)=\text { extensionality }\right) \&\right. \\
& \left.\left(\mathbb{N}, \in_{x}\right) \text { is well-founded of rank } \xi\right\} .
\end{aligned}
$$

Let $F_{\alpha}^{S_{\infty}}$ be the relation of isomorphism on $\bigcup_{\xi<\alpha} A_{\xi}$. It is straightforward, by induction on $\alpha$, to show that $F_{\alpha}^{S_{\infty}}$ is Borel.

If now $(X, a)$ is a Borel $S_{\infty}$-space with $E_{a}$ Borel, then we can assume by 2.7.3 that $X=\operatorname{Mod}(\sigma)$, for some sentence $\sigma \in L_{\omega_{1} \omega}$, where $L$ is as in 2.7.3, and $a=J_{L} \mid X$. Then by 7.1.4, there is an $\alpha<\omega_{1}$ such that $\operatorname{sr}\left(\mathcal{A}_{x}^{L}\right)<\alpha$, 
for $x \in \operatorname{Mod}(\sigma)$. We can then find a Borel function $f: \operatorname{Mod}(\sigma) \rightarrow X_{L_{0}}$ such that $f(x) \in \bigcup_{\xi<\beta} A_{\xi}$, for some $\beta<\omega_{1}$, and $x E_{a} y \Leftrightarrow \mathcal{A}_{f(x)}^{L_{0}} \cong \mathcal{A}_{f(y)}^{L_{0}}$. Here $f(x)$ is simply a "code" of the transitive closure of the canonical Scott sentence of $\mathcal{A}_{x}^{L_{0}}$, i.e., if $\mathcal{A}_{f(x)}^{L_{0}}=\left(\mathbb{N}, \in_{f(x)}\right)$, then the Mostowski collapse of $\mathcal{A}_{f(x)}^{L_{0}}$ is the transitive closure of the canonical Scott sentence of $\mathcal{A}_{x}$. (For the notion of canonical Scott sentence, see Barwise [75].)

Let now $F_{\beta}^{\prime}=F_{\beta}^{S_{\infty}} \times I\left(2^{\mathbb{N}}\right)$, where $I\left(2^{\mathbb{N}}\right)=2^{\mathbb{N}} \times 2^{\mathbb{N}}$, fix a homeomorphism $x \mapsto g(x)$ from $X_{L}$ onto $2^{\mathbb{N}}$ and put $h(x)=(f(x), g(x))$. Then $h$ is injective Borel and

$$
x E_{a} y \Leftrightarrow h(x) F_{\beta}^{\prime} h(y),
$$

i.e., $E_{a}$ is Borel e.r.-embeddable into $F_{\beta}^{\prime}$. Now, easily, $F_{\beta}^{\prime}$ is Borel e.r.embeddable into $F_{\beta+2}^{S_{\infty}}$, thus $\left\{F_{\alpha}^{S_{\infty}}\right\}$ is a universal sequence for $\mathcal{E}_{S_{\infty}}^{B}$.

Remark. Clearly $F_{\alpha}^{S_{\infty}}$ is Borel e.r.-embeddable in $F_{\beta}^{S_{\infty}}$ if $\alpha \leq \beta$. It was shown in Friedman-Stanley [89] that $F_{\beta}^{S_{\infty}}$ is not e.r.-embeddable in $F_{\alpha}^{S_{\infty}}$ if $\alpha<\beta$. Harrington (unpublished) has shown that there is no Borel equivalence relation $E$, such that all $F_{\alpha}^{S_{\infty}}$ are Borel e.r.-embeddable in $E$.

7.3.5 Open problem. Is the sequence $\left\{F_{\alpha}^{S_{\infty}}\right\}$ universal for the class $\mathcal{E}^{B}$ of all Borel equivalence relations induced by Borel actions of Polish groups?

Finally, for further reference, let us note that the proof of 7.3.1 establishes also the following fact.

7.3.6 Theorem. Let $G$ be a Polish group, $X$ a Polish space, $Y \subseteq X$ a $\Pi_{1}^{1}$ set and let $a$ be a Borel-measurable action of $G$ on $Y$. Then there is a sequence $\left\{A_{\xi}\right\}_{\xi<\omega_{1}}$ of pairwise disjoint Borel (in $X$ ) subsets of $Y$ with the following properties:

(i) $A_{\xi}$ is invariant and $\bigcup_{\xi} A_{\xi}=Y$;

(ii) $E_{a} \mid A_{\xi}$ is Borel (in $X^{2}$ );

(iii) there is a $C$-measurable function $f: Y \rightarrow 2^{\mathbb{N} \times \mathbb{N}}$ such that $f(y) \in$ WO and $y \in A_{|f(y)|}$;

(iv) if $A \subseteq Y$ is invariant Borel (in $X$ ) and $E_{a} \mid A$ is Borel (in $X^{2}$ ), then $A \subseteq \bigcup_{\xi<\alpha} A_{\xi}$ for some $\alpha<\omega_{1}$.

\subsection{Tame groups}

Let us call a Polish group $G$ tame if for every Borel $G$-space $(X, a)$ the equivalence relation $E_{a}$ is Borel. By 5.2.1 we can substitute here "Borel $G$-space" by "Polish $G$-space". It is clear that if $G$ is tame and $\varphi: G \rightarrow G^{\prime}$ 
is a continuous surjective homomorphism, then $G^{\prime}$ is tame. By 2.3.5 it also follows that if $G$ is tame and $H \subseteq G$ is a closed subgroup, then $H$ is tame as well. Finally, from 3.5.3, if $G$ is tame and $G \times \mathbb{Z}$ is isomorphic to a (closed) subgroup of $G$, then $G$ is tame iff $E_{a}$ is Borel, where $a$ is the left-action of $G$ on $\mathcal{F}(G)$.

It would be interesting to delineate the class of tame Polish groups. Since the equivalence relation induced by a continuous action of a locally compact Polish group on a Polish space is easily $F_{\sigma}$, it follows that locally compact Polish groups are tame. On the other hand, as we mentioned in $3.2, S_{\infty}$ is not tame. Around 1980 Sami raised the question whether (in our terminology) abelian Polish groups are tame (see Sami [94]). Solecki [9?] answered this in the negative, by studying product groups $\prod_{n \in \mathbb{N}} H_{n}$, with $H_{n}$ countable. In fact, for abelian $H_{n}$ he has obtained a beautiful purely algebraic characterization of the sequences of groups $\left(H_{n}\right)$ for which $\prod_{n} H_{n}$ is tame. In particular, there are such products $\prod_{n} H_{n}$ with $H_{n}$ abelian, which are (nonlocally compact but) tame, although in some sense "most" such products are not tame. The problem of characterizing algebraically the sequences $\left(H_{n}\right)$, with $H_{n}$ countable but not necessarily abelian, for which $\prod_{n} H_{n}$ is tame is still open, but Solecki [9?] makes substantial progress on this question.

As we just mentioned, Solecki [9?] produces examples of $\left(H_{n}\right), H_{n}$ countable abelian, with $\prod_{n} H_{n}$ not tame. In fact $\mathbb{Z}^{\mathbb{N}}$ is such an example. This, as explained in Solecki's paper, is related to the work of Makkai [81], in which an example is given of an $L_{\omega_{1} \omega}$ sentence which has no uncountable model but has countable models of arbitrarily high Scott rank. We would like now to present a general form of Makkai's ideas in the framework of the concepts and results introduced in this book.

First we start with a countable structure $\mathcal{A}_{0}$ such that its canonical Scott sentence, say $\sigma_{\mathcal{A}_{0}}$, has no uncountable model and $G=\operatorname{Aut}\left(\mathcal{A}_{0}\right)$ is not tame. Say $L_{0}$ is the language of $\mathcal{A}_{0}$ and view $\mathcal{A}_{0}$ as having universe $\mathbb{N}$. Let $L$ be a language disjoint from $L_{0}$, with one relation symbol for each arity. By 2.7.4 the relativized logic action $J_{L_{0} \cup L}^{\mathcal{A}_{0}}$ is a universal $\operatorname{Aut}\left(\mathcal{A}_{0}\right)$-action, so its associated equivalence relation is not Borel. It follows that the logic action $J_{L_{0} \cup L}$ restricted to the models of $\sigma_{\mathcal{A}_{0}}$, but with $\sigma_{\mathcal{A}_{0}}$ viewed now as a sentence in $L_{0} \cup L$, has a non-Borel associated equivalence relation, i.e., isomorphism restricted to the $L_{0} \cup L$-structures which model $\sigma_{\mathcal{A}_{0}}$ is not Borel. By 7.1.4 these models must have unbounded Scott ranks. Thus $\sigma_{\mathcal{A}_{0}}$, viewed as a sentence in $L_{0} \cup L$, has countable models of arbitrarily high Scott rank, but no uncountable model.

As an example of such $\mathcal{A}_{0}$ one can take the linear ordering which consists of $\omega$ copies of $\mathbb{Z}$ (ordered like $\omega$ ), whose automorphism group is $\mathbb{Z}^{\mathbb{N}}$. 
We conclude with a descriptive application of Solecki's result that $\mathbb{Z}^{\mathbb{N}}$ is not tame, and a related problem.

Let $G$ be a non-tame Polish group, with $G \times \mathbb{Z}$ isomorphic to a (closed) subgroup of $G$. Then, as we mentioned in the beginning of 7.4, the left action of $G$ on $\mathcal{F}(G)$ gives rise to a non-Borel equivalence relation. This means that the equivalence relation, among closed subsets of $\mathcal{F}(G)$, which is defined by

$$
F \sim F^{\prime} \Leftrightarrow F \text { is a left-translate of } F^{\prime}
$$

is $\boldsymbol{\Sigma}_{1}^{1}$ but not Borel. In particular, since $\mathbb{Z}^{\mathbb{N}}$ is a closed subgroup of $\mathbb{R}^{\mathbb{N}}$ and so $\mathbb{R}^{\mathbb{N}}$ is not tame, it follows that the equivalence relation of "being a translate of" among closed subsets of $\mathbb{R}^{\mathbb{N}}$ is not Borel. As far as we know though, it is open whether the same holds for $\left(\ell^{2},+\right)$, i.e., whether $\left(\ell^{2},+\right)$ is not tame.

\subsection{Normalizers}

We consider finally the conjugation action $c$ of a Polish group $G$ on the set of its closed subgroups $\mathcal{S}(G)$, and the corresponding equivalence relation $E_{c}^{G}=E_{c}$. As opposed to the left action of $G$ on $\mathcal{F}(G)$, we do not know whether the following holds: $G$ is tame iff $E_{c}$ is Borel (at least for $G$ such that $G \times \mathbb{Z}$ is isomorphic to a (closed) subgroup of $G$ ). Notice that the stabilizer of a point $F \in \mathcal{S}(G)$ under this action is the normalizer $N_{G}(F)=\left\{g \in G: g F g^{-1}=F\right\}$ of $F$ in $G$. So the question of whether $E_{c}$ is Borel is equivalent to the question of whether $F \mapsto N_{G}(F)$ is Borel, by 7.1.2. We can answer this question for $G=S_{\infty}$.

7.5.1 Theorem. The equivalence relation of conjugacy among closed subgroups of $S_{\infty}$ is not Borel. Equivalently, the function $F \mapsto N_{S_{\infty}}(F)$ (has co-analytic graph but) is not Borel (on the standard Borel space of closed subgroups of $S_{\infty}$ ).

Proof. Let $L_{0}$ be the language consisting of a single binary relation symbol and $X \subseteq X_{L_{0}}$ the set of $x \in X_{L_{0}}$ for which $\mathcal{A}_{x}$ is a Boolean algebra.

Recall that a superatomic Boolean algebra is one all of whose subalgebras are atomic. The countable superatomic infinite Boolean algebras are, up to isomorphism, exactly the interval algebras of the wellorderings $\omega^{\rho} k(0<$ $\left.\rho<\omega_{1}, 1 \leq k<\omega\right)$; see Monk [89, p. 277].

Let $\mathrm{SA} \subseteq X$ be the set of $x \in X$ for which $\mathcal{A}_{x}$ is superatomic. Then SA is clearly $\Pi_{1}^{1}$ (effectively co-analytic). Moreover, by the above, there is a $\Delta_{2}^{1}$ function $\varphi: \mathrm{SA} \rightarrow$ WO with

$$
\mathcal{A}_{x} \simeq \mathcal{A}_{y} \Leftrightarrow|\varphi(x)|=|\varphi(y)| .
$$


(Actually, $|\varphi(x)|$ is a $\Pi_{1}^{1}$-rank but we will not need this here.) By a theorem of McKenzie [77], for $x, y \in \mathrm{SA}$ :

$$
\mathcal{A}_{x} \cong \mathcal{A}_{y} \Leftrightarrow \operatorname{Aut}\left(\mathcal{A}_{x}\right) \text { is algebraically isomorphic to } \operatorname{Aut}\left(\mathcal{A}_{\mathrm{y}}\right) .
$$

But, trivially,

$$
\begin{aligned}
\mathcal{A}_{x} \cong \mathcal{A}_{y} & \Rightarrow \operatorname{Aut}\left(\mathcal{A}_{x}\right) E_{c} \operatorname{Aut}\left(\mathcal{A}_{y}\right) \\
& \Rightarrow \operatorname{Aut}\left(\mathcal{A}_{x}\right) \text { is algebraically isomorphic to } \operatorname{Aut}\left(\mathcal{A}_{y}\right) .
\end{aligned}
$$

So for $x, y \in \mathrm{SA}$,

$$
\mathcal{A}_{x} \cong \mathcal{A}_{y} \Leftrightarrow \operatorname{Aut}\left(\mathcal{A}_{x}\right) E_{c} \operatorname{Aut}\left(\mathcal{A}_{y}\right)
$$

Let

$$
A=\left\{\operatorname{Aut}\left(\mathcal{A}_{x}\right): x \in \mathrm{SA}\right\} .
$$

Assuming strong set theoretic axioms, it is now obvious that the equivalence relation of conjugacy is not Borel. Roughly speaking, strong axioms imply that for any $\alpha<\omega_{1}$, there is no "definable" way to produce a collection of exactly $\aleph_{1} \Pi_{\alpha}^{0}$ sets, i.e., there is no way to do it without using AC. This is a theorem of Harrington [78], which has several precise versions. One version is: Assuming PD (see $\S 5.3$ ), there is no projective $\omega_{1}$-sequence of distinct $\Pi_{\alpha}^{0}$ sets, that is, no injection $\Phi: \omega_{1} \rightarrow \mathcal{P}(\mathbb{R})$, with range $(\Phi)$ consisting of $\Pi_{\alpha}^{0}$ sets such that $\Phi$ is projective in the codes. Clearly $A$ contains exactly $\aleph_{1}$ conjugacy classes, and, in fact, it is easy to see that we get a projective $\omega_{1}$-sequence of classes. So assuming $\mathrm{PD}$, the conjugacy classes are not of bounded Borel rank, hence the equivalence relation of conjugacy is not Borel.

Unfortunately, this transparent argument requires strong axioms. The proof in ZFC - which we give below - is much less straightforward and involves forcing.

It will now be convenient to set up a system of coding closed subgroups of $S_{\infty}$ by members of the Baire space $\mathcal{N}$. For $x \in \mathcal{N}$, let $(x)_{n} \in \mathcal{N}$ be defined by

$$
(x)_{n}(m)=x(\langle n, m\rangle),
$$

where $\langle n, m\rangle$ is a recursive bijection of $\mathbb{N} \times \mathbb{N}$ with $\mathbb{N}$. Put

$$
C=\left\{x \in \mathcal{N}: \forall n\left((x)_{n} \in S_{\infty}\right) \&\left\{(x)_{n}: n \in \mathbb{N}\right\} \text { is a subgroup of } S_{\infty}\right\} .
$$

Then $C$ is a $\Delta_{1}^{1}$ subset of $\mathcal{N}$. For $x \in C$, let

$$
\pi(x)=\text { the closure of }\left\{(x)_{n}: n \in \mathbb{N}\right\} \text { in } S_{\infty} .
$$


Thus $\pi: C \rightarrow \mathcal{S}\left(S_{\infty}\right)$ is a Borel surjection. For $x, y \in C$, put

$$
x E_{c}^{*} y \Leftrightarrow \pi(x) E_{c} \pi(y) .
$$

Then it is easy to check that $E_{c}^{*}$ is a $\Sigma_{1}^{1}$ equivalence relation on $C$. Let

$$
A^{*}=\{x \in C: \pi(x) \in A\} \text {. }
$$

Again it is easy to see that $A^{*}$ is $\Sigma_{2}^{1}$.

Assume now, towards a contradiction, that $E_{c}$ is Borel. Then clearly $E_{c}^{*}$ is Borel, so fix a parameter $p \in \mathcal{N}$ such that $E_{c}^{*}$ is $\Delta_{1}^{1}(p)$. Consider a generic extension $V[G]$ of the universe $V$ in which $\aleph_{\omega_{1}^{p}}^{L[p]}<\aleph_{1}$ and $\mathrm{MA}_{\aleph_{1}}$ holds. (Here $\omega_{1}^{p}$ is the first non-recursive in $p$ ordinal.) Then, in $V[G], E_{c}^{*}$ is still $\Delta_{1}^{1}(p)$, by Shoenfield absoluteness, and by $\mathrm{MA}_{\aleph_{1}}$ every $\boldsymbol{\Sigma}_{2}^{1}$ set is Lebesgue measurable. We work in $V[G]$ from now on. The set $A^{*}$ is clearly an $E_{c}^{*}$-invariant subset of $C$ and clearly contains more than countably many $E_{c}^{*}$-equivalence classes. Since $\aleph_{\omega_{1}^{p}}^{L[p]}<\aleph_{1}$ and $E_{c}^{*}$ is $\Delta_{1}^{1}(p)$, by a result of Stern [84, 9.3], it follows that there is a Cantor set $P \subseteq A^{*}$, any two elements of which are not $E_{c}^{*}$-equivalent. There is clearly a $\Delta_{2}^{1}$ function $f: P \rightarrow \mathrm{SA}$ such that for $x \in P, f(x)=y$ with $\pi(x)=\operatorname{Aut}\left(\mathcal{A}_{y}\right)$. Then $|\varphi \circ f|: P \rightarrow \omega_{1}$ is one-to-one and thus the relation

$$
x<y \text { iff }|\varphi(f(x))|<|\varphi(f(y))|
$$

is a $\boldsymbol{\Delta}_{2}^{1}$ wellordering of $P$. Thus $2^{\mathbb{N}}$ admits a $\boldsymbol{\Delta}_{2}^{1}$ wellordering, which is therefore non-measurable, violating $\mathrm{MA}_{\aleph_{1}}$.

In Sami [94], it is essentially shown that if $G$ is a Polish group, $X$ is a Polish $G$-space, and $x \mapsto G_{x}$ is constant on each orbit, then TVC2 holds for this action. We note here the following curious fact that goes in the opposite direction.

7.5.2 Theorem. Assume $\forall x \in 2^{\mathbb{N}}\left(\aleph_{1}^{L[x]}<\aleph_{1}\right)$. Let $G$ be a Polish group, for which $E_{c}^{G}$ is Borel, $(X, a)$ a Borel $G$-space, and assume $x \mapsto G_{x}$ is one-to-one on each orbit. If $A \subseteq X$ is $\Sigma_{2}^{1}$ and invariant, either $A$ contains countably many orbits or there is a Cantor set $C \subseteq A$ any two distinct elements of which belong to different orbits.

Proof. Consider the space $X \times \mathcal{S}(G)$ and the action $b(g,(x, F))=(g$. $\left.x, g F g^{-1}\right)$ of $G$ on it. Let $Y \subseteq X \times \mathcal{S}(G)$ be given by

$$
(x, F) \in Y \Leftrightarrow F \subseteq G_{x} \& F=N_{G}(F) .
$$


Then $Y$ is invariant under this action. Also $Y$ is Borel, since $F \mapsto N_{G}(F)$ is Borel. Moreover, for $(x, F) \in Y$ the stabilizer $G_{(x, F)}$ is given by

$$
G_{(x, F)}=G_{x} \cap N_{G}(F)=G_{x} \cap F=F .
$$

So $(x, F) \in Y \mapsto G_{(x, F)}$ is obviously Borel and thus $E_{b} \mid Y$ is Borel as well. Let $i: X \rightarrow X \times \mathcal{S}(G)$ be defined by

$$
i(x)=\left(x, G_{x}\right) .
$$

Our assumption that $x \mapsto G_{x}$ is one-to-one on each orbit of $X$ is equivalent to the fact that $N_{G}\left(G_{x}\right)=G_{x}$, so $i: X \rightarrow Y$. Also

$$
x E_{a} y \Leftrightarrow i(x) E_{b} i(y) .
$$

Let now $A \subseteq X$ be $\boldsymbol{\Sigma}_{2}^{1}$ and invariant. Then $i(A) \subseteq Y$ is $\boldsymbol{\Sigma}_{2}^{1}$ and invariant. Since $E_{b} \mid Y$ is Borel, by a theorem of Stern [84, §9], it follows that $i(A)$ contains countably many orbits or there is a Cantor subset of it any two elements of which belong to distinct orbits. From this it easily follows that the same holds for $A$.

In the preceding proof we have not really used that $E_{c}^{G}$ is Borel, but only that the set $\left\{F \in \mathcal{S}(G): F=N_{G}(F)\right\}$ of self-normalizing subgroups of $G$ is Borel. Concerning $S_{\infty}$ we can also show, using some examples of $\mathrm{S}$. Thomas (private communication), that this set is not Borel, which is a strengthening of 7.5.1.

7.5.3 Theorem. The set $\left\{F \in \mathcal{S}\left(S_{\infty}\right): F=N_{S_{\infty}}(F)\right\}$ of self-normalizing closed subgroups of $S_{\infty}$ is (co-analytic but) not Borel.

Proof. As in the proof of 7.5.1, we show that if this theorem is false then we can produce a definable collection of $\aleph_{1}$ Borel sets of bounded rank. Also as in the proof of 7.5.1, we then go through some contortions to make the proof work in ZFC. For each countable field $K$, let $P G(4, K)$ be the associated 4-dimensional projective space and consider the structure $\mathcal{M}_{K}=\langle P G(4, K), Q\rangle$, where $Q$ is the ternary relation of collinearity. S. Thomas (private communication) shows that $\operatorname{Aut}\left(\mathcal{M}_{K}\right)$ is equal to its own normalizer (in the symmetric group of its universe). Moreover, by standard results in projective geometry,

$$
K \cong K^{\prime} \Leftrightarrow \mathcal{M}_{K} \cong \mathcal{M}_{K^{\prime}}
$$

We next use a special case of a result of Friedman-Stanley [89], according to which there is a $\Delta_{1}^{1} \operatorname{map} F: X_{L_{0}} \rightarrow X_{L_{1}}$, where $L_{0}$ is the language of 
linear orderings, and $L_{1}$ is the language of fields (viewed as relational), so that for $x \in X_{L_{0}}$ with $\mathcal{A}_{x}^{L_{0}}$ a linear ordering, $\mathcal{A}_{F(x)}^{L_{1}}$ is a field and

$$
\mathcal{A}_{x}^{L_{0}} \cong \mathcal{A}_{y}^{L_{0}} \Leftrightarrow \mathcal{A}_{F(x)}^{L_{1}} \cong \mathcal{A}_{F(y)}^{L_{1}} \text {. }
$$

Also let $L_{2}$ be the language with a ternary relation symbol and $G$ : $X_{L_{1}} \rightarrow X_{L_{2}}$ a $\Delta_{1}^{1}$ map such that if $K=\mathcal{A}_{x}^{L_{1}}$ is a field, then $\mathcal{A}_{G(x)}^{L_{2}} \cong \mathcal{M}_{K}$. So if $P=G \circ F$, then for $x, y \in X_{L_{0}}$ with $\mathcal{A}_{x}, \mathcal{A}_{y}$ linear orderings, we have

$$
\mathcal{A}_{x}^{L_{0}} \cong \mathcal{A}_{y}^{L_{0}} \Leftrightarrow \mathcal{A}_{P(x)}^{L_{2}} \cong \mathcal{A}_{P(y)}^{L_{2}} \text {. }
$$

Now let

$$
R=\left\{y \in X_{L_{2}}: \exists x\left(x \in \mathrm{WO}^{*} \& P(x)=y\right)\right\} .
$$

Then $R$ is $\Sigma_{2}^{1}$ in $X_{L_{2}}$. For $y \in R$, let $\varphi(y)=|x|$, where $x \in \mathrm{WO}^{*}$ is such that $P(x)=y$ (note that this $|x|$ is uniquely determined). Then $\varphi: R \rightarrow \omega_{1} \backslash \omega$ is a surjection, and for $y, y^{\prime} \in R$,

$$
\mathcal{A}_{y}^{L_{2}} \cong \mathcal{A}_{y^{\prime}}^{L_{2}} \Leftrightarrow \varphi(y)=\varphi\left(y^{\prime}\right),
$$

and the relation $\varphi(y) \leq \varphi\left(y^{\prime}\right)$ on $R$ is $\Sigma_{2}^{1}$. Finally, $y \in R$ implies that $\operatorname{Aut}\left(\mathcal{A}_{y}\right)$ is self-normalizing.

Now consider the logic action $a=J_{L_{2}}$ of $S_{\infty}$ on $X_{L_{2}}$ and the set $Y \subseteq$ $X_{L_{2}} \times \mathcal{S}\left(S_{\infty}\right)$ as defined in 7.5.2 for this action. Using the coding $\pi: C \rightarrow$ $\mathcal{S}\left(S_{\infty}\right)$ as in the proof of 7.5 .1 , we see that $Y^{*}=\left\{(x, y) \in X_{L_{2}} \times C\right.$ : $(x, \pi(y)) \in Y\}$ is $\Pi_{1}^{1}$.

Now assume $\left\{F \in \mathcal{S}\left(S_{\infty}\right): F=N_{S_{\infty}}(F)\right\}$ is Borel, so that $Y$ is Borel too. Considering the action $b$ of $S_{\infty}$ on $X_{L_{2}} \times \mathcal{S}\left(S_{\infty}\right)$ as in the proof of 7.5.2 again, we see that $E_{b} \mid Y$ is Borel too. For $(x, y),\left(x^{\prime}, y^{\prime}\right) \in X_{L_{2}} \times C$ put

$$
(x, y) E_{b}^{*}\left(x^{\prime}, y^{\prime}\right) \Leftrightarrow(x, \pi(y)) E_{b}\left(x^{\prime}, \pi\left(y^{\prime}\right)\right) .
$$

Then $E_{b}^{*}$ is $\Sigma_{1}^{1}$. Since $Y, E_{b} \mid Y$ are Borel, clearly $Y^{*}$ and $E_{b}^{*} \mid Y^{*}$ are Borel. So fix a parameter $p \in \mathcal{N}$ so that both are $\Delta_{1}^{1}(p)$. Let $V[G]$ be a generic extension of the universe $V$ in which $\aleph_{\omega_{1}^{p}}^{L[p]}<\aleph_{1}$ and $M A_{\aleph_{1}}$ holds. Then, working in $V[G]$ from now on, we have that $Y^{*}, E_{b}^{*} \mid Y^{*}$ are still $\Delta_{1}^{1}(p)$ by Shoenfield absoluteness. Also if

$$
i(R)=\left\{\left(x, \operatorname{Aut}\left(\mathcal{A}_{x}\right)\right): x \in R\right\},
$$


and

$$
i(R)^{*}=\left\{(x, y) \in X_{L_{2}} \times C:(x, \pi(y)) \in i(R)\right\},
$$

then $i(R)^{*}$ is a $\Sigma_{2}^{1}$ subset of $Y^{*}$ which is $E_{b}^{*}$-invariant. For $(x, y),\left(x^{\prime}, y^{\prime}\right) \in$ $i(R)^{*}$, let

$$
(x, y) \leq^{*}\left(x^{\prime}, y^{\prime}\right) \Leftrightarrow \varphi(x) \leq \varphi\left(x^{\prime}\right) .
$$

Then $\leq^{*}$ is a $\Sigma_{2}^{1}$ prewellordering on $i(R)^{*}$ of length exactly $\omega_{1}$, and

$$
(x, y) E_{b}^{*}\left(x^{\prime}, y^{\prime}\right) \Leftrightarrow \varphi(x)=\varphi\left(x^{\prime}\right)
$$

So $E_{b}^{*} \mid i(R)^{*}$ has exactly $\aleph_{1}$ classes, and as in the proof of 7.5 .1 we get a contradiction. 